\title{
Movimientos de roedores intra- e inter-ambiente y riesgo de exposición al Hantavirus "Andes" en Patagonia norte, Argentina
}

\author{
Martín J. Monteverde ${ }^{1, \mathbb{}}$ \& Karina Hodara ${ }^{2}$ \\ ${ }^{1}$ Dirección de Ecosistemas Terrestres, Centro de Ecología Aplicada del Neuquén, Junín de los Andes, Neuquén, Argentina. \\ ${ }^{2}$ Departamento Métodos Cuantitativos y Sistemas de Información, Facultad de Agronomía, Universidad de Buenos Aires, \\ Buenos Aires, Argentina.
}

\begin{abstract}
Resumen. Se estudiaron algunos aspectos de la ecología espacial de tres especies de roedores sigmodontinos (Oligoryzomys longicaudatus, Abrothrix olivacea y Abrothrix hirta) en el Paraje El Contra del Parque Nacional Lanín (Provincia de Neuquén, Argentina). Los resultados se analizaron en el marco del riesgo de exposición humana al Hantavirus "Andes", agente causal del Síndrome Pulmonar por Hantavirus. El trabajo se realizó en dos tipos de ambiente: silvestre y doméstico. Los roedores fueron capturados mensualmente con la metodología de captura-marcado-recaptura. De esta manera se estimaron y compararon los movimientos dentro y entre ambos ambientes y los tamaños de áreas de acción. Oligoryzomys longicaudatus, principal reservorio del Hantavirus "Andes" en Patagonia, presentó el mayor número de capturas en ambos ambientes y más de lo esperado por azar dentro de los ambientes domésticos. Además, mostró las áreas de acción con menor tamaño promedio y movimientos intra-ambiente estadísticamente significativos. Los ambientes domésticos rurales resultaron ser potencialmente los más riesgosos en términos de exposición y transmisión de Hantavirus a humanos. Como resultado de las capturas y movimientos dispersivos de O. longicaudatus, se discuten otras contribuciones de este trabajo a la salud humana.
\end{abstract}

[Palabras clave: dispersión, ambientes domésticos y silvestres, Oligoryzomys longicaudatus, Parque Nacional Lanín, sigmodontinae, uso de hábitat, zoonosis]

\begin{abstract}
Aвstract. Intra- and inter-environment movements of rodents and risk of exposure to "Andes" Hantavirus in northern Patagonia, Argentina. Some aspects of the spatial ecology of three species of sigmodontine rodents (Oligoryzomys longicaudatus, Abrothrix olivacea and Abrothrix hirta) were studied in the Paraje El Contra, Lanín National Park (Province of Neuquén, Argentina), and the results were then analyzed in relation to the risk of human exposure to "Andes" Hantavirus, causative agent of Hantavirus Pulmonary Syndrome. The work was conducted in two environment types (sylvan and domestic), where mice were monthly captured using capture-mark-recapture methodology. Thus, movements within and between environments and home ranges sizes were estimated and compared. Oligoryzomys longicaudatus, principal reservoir of "Andes" Hantavirus in Patagonia, showed the largest number of captures in both environments and more captures than those expected by random within domestic environments. Moreover, it showed the lowest average home range sizes, and statistically significant intra environment movements. Rural domestic environments resulted to be the riskiest in terms of exposure and transmission of Hantavirus to humans. Other contributions of this work to human health are discussed as a result of captures and dispersal movements of $O$. longicaudatus.
\end{abstract}

[Keywords: dispersal, domestic and sylvan environments, Oligoryzomys longicaudatus, Lanín National Park, sigmodontinae, habitat use, zoonosis]

\section{INTRODUCCIÓN}

Conocer la organización de los animales en el espacio y en el tiempo es central en ecología. La dinámica poblacional de una especie está muy ligada a su organización espacial y a los movimientos de sus individuos, causados por presiones internas o externas a esa población (Kernohan et al. 2001). Por lo tanto, el uso del espacio por individuos de diferentes especies de animales es el resultado de una combinación de factores internos (e.g., su fisiología y morfología), factores externos (e.g., el ambiente en el que se encuentran) (Giuggioli et al. 2006) y relaciones interespecíficas de competencia y depredación (Harris and Macdonald 2007).

Los movimientos dispersivos en los animales se asocian a la adquisición de recursos, a evitar depredación y competencia, y a comportamientos de apareamiento (Fahrig 2007). Así, los animales ocuparán en primer lugar los hábitats más favorables o de mayor calidad de una manera eficiente. En pequeños mamíferos, la disponibilidad de recursos y el riesgo de depredación influyen en gran medida la calidad del hábitat (Morris 1987; Lin and Batzli 2001). Por lo tanto, el éxito de localizar hábitatsfavorables dependerá deuna estrategia

Editor asociado: Sergio Lambertucci 
óptima de búsqueda, del grado de percepción de hábitats cualitativamente óptimos y de los costos de la dispersión (configuración espacial de los hábitats, distancia entre ellos, o riesgo de depredación en la dispersión) (Zollner 2000). Los costos y beneficios de dispersión varían en el tiempo y en el espacio entre los individuos de cada especie (Bowler and Benton 2005). El principal beneficio es evitar la competencia intraespecífica en el sitio actual y ocupar hábitats subexplotados, mientras que el principal costo es la mortalidad durante ese movimiento dispersivo (Morris 1989, 1991). En consecuencia, el balance será reflejado en el éxito reproductivo individual en respuesta a la decisión de dispersarse (Moenting and Morris 2006).

La dispersión entre parches en un ambiente heterogéneo es clave en la estructuración de las poblaciones animales en esos ambientes (Zheng et al. 2009). En este sentido la fragmentación y la pérdida de hábitat impactan sobre procesos ecológicos asociados, y esto afecta características demográficas y movimientos entre las poblaciones (Pavlacky et al. 2012). Por tal motivo, obtener mejor información sobre los factores ambientales que determinan los movimientos de los individuos es un componente crucial de modelos predictivos de la dispersión y selección de hábitat individual en paisajes heterogéneos (Avgar et al. 2012).

Oligoryzomys longicaudatus, Abrothrix hirta (denominado $A$. longipilis previo a Teta and Pardiñas 2014) y Abrothrix olivacea (Rodentia, Cricetidae, Sigmodontinae) son las tres especies silvestres más abundantes que componen el ensamble de roedores sigmodontinos en los ambientes boscosos de Patagonia norte (provincias de Neuquén y Río Negro) (Pearson 1983; Monjeau et al. 1997; Kelt 2000; Udrizar Sauthier et al. 2005) y las más frecuentemente capturadas en dichos sistemas de la Provincia de Neuquén (Piudo et al. 2005; Piudo 2011; Monteverde 2014). Una de estas especies, O. longicaudatus, es el principal reservorio natural del virus "Andes Sur" (ANDV, agente etiológico del Síndrome Pulmonar por Hantavirus) en Patagonia (López et al. 1996; Levis et al. 1998), aunque también se han detectado anticuerpos contra ANDV en las otras 2 especies del ensamble (Larrieu et al. 2003, 2008; Piudo et al. 2005). En Neuquén se diagnosticaron 51 casos de Síndrome Pulmonar por Hantavirus, con $40 \%$ de mortalidad entre los años 1997 y febrero 2017 (Med. María Fernanda
Hadad, Subsecretaría de Salud de Neuquén, comunicación personal). En la mayoría de los casos, los sitios de contagio y exposición al virus fueron las áreas rurales y semi-rurales, en donde pueden distinguirse dos tipos de ambientes, el doméstico y el silvestre. Según algunos autores, $O$. longicaudatus presenta una movilidad alta como característica demográfica que la diferencia de las otras dos especies con las que habita en simpatría (Murúa et al. 1986; Kelt et al. 1994; Meserve et al. 1999). En este sentido, avanzar en el conocimiento de los movimientos dispersivos realizados por O. longicaudatus, A. hirta y A. olivacea en un ambiente antropizado y fragmentado en ambientes silvestres y domésticos aportará al entendimiento de la dinámica de las poblaciones reservorio y a la epidemiología del Hantavirus. Los objetivos de este estudio fueron evaluar los movimientos dispersivos de individuos deestas tres especies de roedores del ensamble en ambientes silvestre y doméstico y relacionar esta información con el riesgo de infección por ANDV. Hipotetizamos que el uso diferencial de los ambientes disponibles por O. longicaudatus favorece la circulación del ANDV en el paisaje como resultado de su capacidad dispersiva entre ambientes. En este sentido, esperamos mayor uso del ambiente doméstico por individuos de $O$. longicaudatus, mayor número de movimientos entre ambientes y en consecuencia, mayor probabilidad de riesgo de infección por Hantavirus para los seres humanos.

\section{Materiales y Métodos}

\section{Área de estudio}

El área de estudio se encuentra dentro del Parque Nacional Lanín, cuyas 412000 ha se extienden desde los $39.2^{\circ}$ hasta los $40.3^{\circ} \mathrm{S}$ en la Provincia de Neuquén. El sitio específico de estudio fue el Paraje El Contra $\left(39^{\circ} 46^{\prime} 47.1^{\prime \prime} \mathrm{S}-71^{\circ} 22^{\prime} 8.8^{\prime \prime} \mathrm{O}\right.$, margen sur del Lago Huechulafquen, Departamento Huiliches, Provincia de Neuquén) (Figura 1a). El sitio está ubicado dentro de la Provincia Fitogeográfica del Bosque Subantártico, en el Distrito de Bosque Caducifolio. El clima es templado y húmedo con una temperatura media anual de $9.5^{\circ} \mathrm{C}$ en la sección norte y de $5.4^{\circ} \mathrm{C}$ en la sección sur. Las precipitaciones se distribuyen de forma homogénea durante todo el año; en algunos sitios superan los 4000 $\mathrm{mm} / \mathrm{año}$. El tipo de vegetación dominante es el bosque, caducifolio o perennifolio, pero también se hallan grandes praderas 
(Cabrera and Willink 1980). Los ambientes domésticos rurales (hábitats domiciliarios y peridomiciliarios) del Paraje El Contra surgen a partir de modificaciones antrópicas sobre el ambiente boscoso original. Dentro de las perturbaciones antrópicas se incluye la remoción de la vegetación para la construcción de casas, leñeras, letrinas, galpones, depósitos de materiales (e.g., fardos, herramientas, etc.) y la creación de áreas periféricas para potreros, corrales, cuadros de pastoreo, invernaderos, huertas y zonas de acumulación de basura y desperdicios.

\section{Diseño de unidades experimentales}

El diseño del ensayo consistió en un sistema de ocho unidades experimentales independientes, cada una conformada por dos ambientes contrastantes: silvestre y doméstico. Para simular el ambiente doméstico se construyeron ocho unidades de galpones

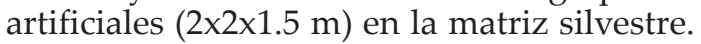
Cada unidad quedó constituida por un ambiente doméstico, con dos compartimentos (viviendas) construidos en su interior, un área de transición y un ambiente silvestre. El área de transición simuló el ecotono (o interfaz) entre un ambiente utilizado por el ser humano y el ambiente silvestre; rodeó por completo el ambiente doméstico y no se realizó trampeo de roedores en esa área. A partir del perímetro externo del área de transición se colocaron seis líneas de cinco trampas cada una (30 trampas en total, separadas por $\sim 10 \mathrm{~m}$ ), en diferentes direcciones de acuerdo a la topografía del lugar en el ambiente silvestre. Las trampas de cada una de estas líneas fueron dispuestas en el terreno con un diseño en "tela de araña" (Parmenter and MacMahon 1989; Parmenter et al. 2003) (Figura 1b).

\section{Trampeo y procesamiento de roedores}

Se realizaron 20 sesiones mensuales de trampeo de roedores entre junio de 2007 y enero de 2010. Se usaron trampas de captura viva tipo Sherman $(8 \times 9 \times 23 \mathrm{~cm}$, HB Sherman Trap Co., Tallahassee, Florida, EE.UU.), activas durante tres noches consecutivas en cada sesión de muestreo. Dichas trampas fueron cebadas con una mezcla de avena arrollada, grasa vacuna y esencia de vainilla. Durante los meses invernales, las trampas fueron provistas de un trozo de algodón para evitar mortalidad por hipotermia de los individuos capturados. Las trampas fueron revisadas cada mañana y se reemplazó el cebo y el algodón si era necesario. El manipuleo y procesamiento de los roedores se realizó siguiendo los lineamientos de bioseguridad estandarizados descriptos en Mills et al. (1995) y Kelt et al. (2010) debido a que el área de muestreo es endémica de Hantavirus. A
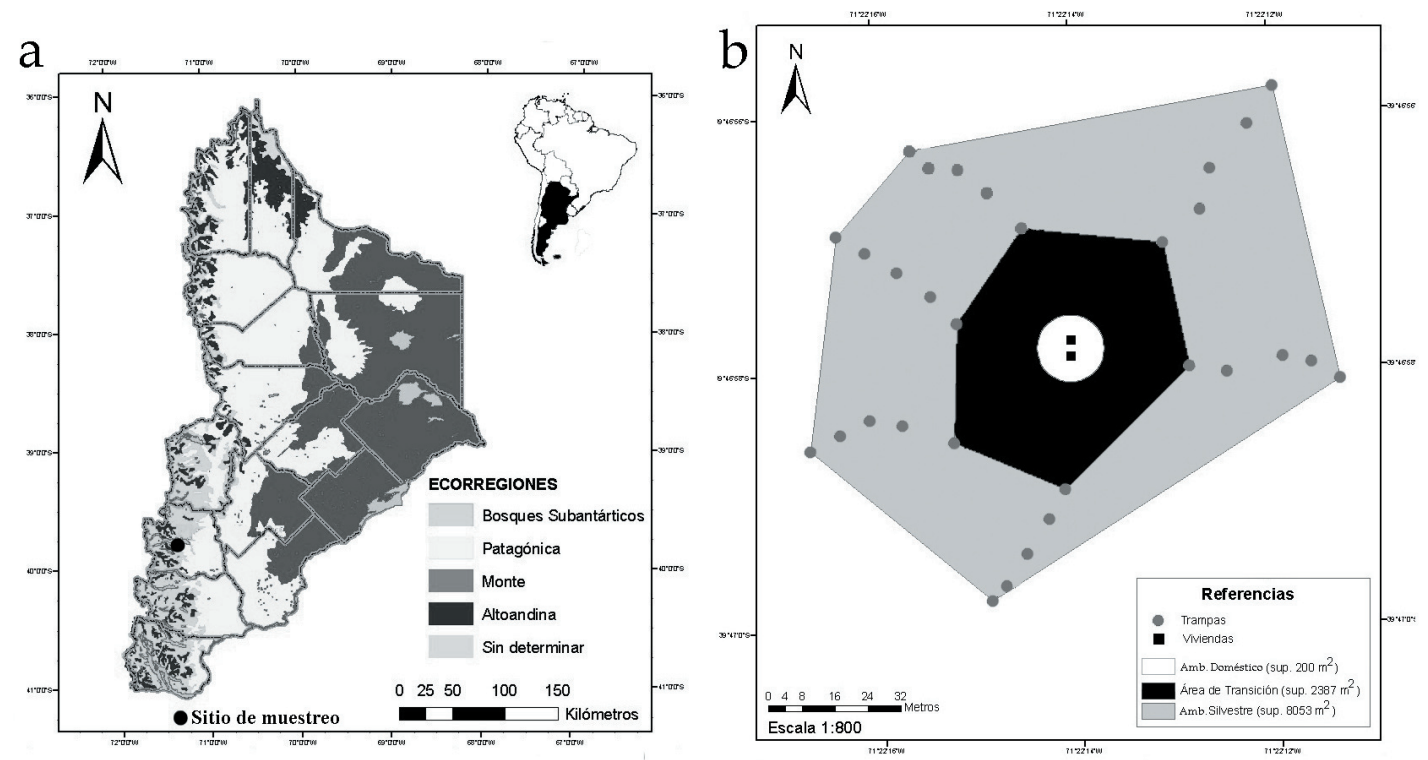

Figura 1. a) Localización geográfica del sitio de muestreo (Paraje El Contra) en la Provincia de Neuquén, con sus ecorregiones, y b) diseño de una de las unidades experimentales, con la disposición de las estaciones de trampeo, los ambientes (silvestre y doméstico), el área de transición y sus respectivas superficies.

Figure 1. a) Geographic location of the sampling site (Paraje El Contra) in Neuquén province, with its ecoregions, and b) one of the experimental units' design, with the arrangement of the trapping stations, environments (sylvan and domestic), the transition area, and their respective areas. 
cada roedor capturado se lo individualizó con una caravana metálica numerada (National Band and Tag Co., Newport, Kentucky, EE.UU.), se le registraron varios parámetros exomorfométricos (i.e., sexo, edad, masa corporal y estado reproductivo) y luego se lo liberó en el mismo sitio de la captura.

\section{Estimación de áreas de acción}

Las áreas de acción fueron estimadas utilizando el método del Mínimo Polígono Convexo con inclusión de borde para roedores adultos capturados y recapturados de $O$. longicaudatus, A. hirta y A. olivacea al menos tres veces durante cada evento de trampeo (Southwood 1966; Corbalán and Ojeda 2005; Monteverde et al. 2011). Estas áreas de acción se consideraron como el área mínima que utiliza cada individuo y se tuvo en cuenta sólo la dimensión horizontal de dichas áreas. Los sitios donde fueron capturados los roedores utilizados para el análisis fueron localizados usando el software ArcGIS 10 (ESRI 2011).

\section{Evaluación del uso de ambientes}

Los patrones de uso de ambientes por las especies de roedores del ensamble fueron evaluados mediante un modelo lineal generalizado mixto cuya variable respuesta es el número de capturas (GLMM, con una distribución Poisson de los errores). Los modelos con distribución Poisson asumen que la media y la varianza de la distribución coinciden. Sin embargo, como los datos obtenidos presentaron sobredispersión (varianza de la variable respuesta mayor que la media [Hilbe 2011]), se incluyó al GLMM un efecto aleatorio con un único nivel para cada dato particular (OLRE, observationlevel random effects) (Harrison 2014) para corregir dicha sobredispersión. De esta manera, el modelo resultó con dos factores principales fijos: ambiente (doméstico y silvestre) y especie (tres niveles) y dos factores aleatorios: sitio y OLRE. El análisis se llevó a cabo usando la función glmer (paquete lme4, R 3.0.3) (Development Core Team 2014). El área de acción de los individuos según su especie y sexo fueron evaluadas mediante un modelo lineal general mixto (GLMM, usando la función lme del paquete nlme, R 3.0.3) (Development Core Team 2014). El mejor modelo incluyó dos factores principales fijos: especie $(O$. longicaudatus y $A$. hirta, debido al insuficiente número de áreas de acción calculadas para $A$. olivacea $[\mathrm{n}=4]$ ) y sexo (dos niveles). El sitio fue incluido como factor aleatorio y el término de interacción entre especie y sexo fue eliminado del modelo resultante. En cuanto al cumplimiento de los supuestos para este modelo, las varianzas resultaron homogéneas; sin embargo, los errores no se distribuían normalmente. Para lograr el cumplimiento de este último supuesto, la variable original fue transformada usando ln (área de acción). Al detectar efectos significativos para algún término de los modelos resultantes se realizaron pruebas post-hoc (LSD de Fisher). Se utilizó el criterio de información de Akaike (AIC) (Burnham and Anderson 2002) para identificar, en cada caso, el modelo que explicara la mayor variación en la variable respuesta con la menor cantidad de parámetros a estimar. De esta manera, los modelos seleccionados fueron aquellos que presentaron menor valor de AIC. Las proporciones de capturas en los domicilios de las viviendas con respecto al resto del ambiente fueron comparadas entre especies con una prueba de homogeneidad de chi cuadrado $\left(\chi^{2}\right)$. Los movimientos de individuos de cada especie intra- e inter-ambiente fueron evaluados usando pruebas exactas de Fisher (Agresti 2002), bajo la hipótesis nula de que el ambiente donde se registraron las capturas originales es independiente del ambiente donde se registraron las capturas posteriores. En este último caso, cada especie de roedor fue analizada por separado. Todas las pruebas que presentaron valores de $P<0.05$ fueron consideradas como estadísticamente significativas.

\section{Resultados}

\section{Captura de roedores y uso de ambientes}

Se capturaron 805 individuos totales pertenecientes a ochoespecies(O.longicaudatus, A. hirta, A. olivacea, Loxodontomys micropus, Chelemys macronyx, Geoxus valdivianus, Irenomys tarsalis y Rattus rattus), los que fueron capturados 1623 veces en 7200 trampas/noche. Oligoryzomys longicaudatus fue el roedor más frecuentemente capturado $(48.07 \%)$, seguido por $A$. hirta con $34.78 \%$ y por A. olivacea con $11.93 \%$, lo que totaliza $94.78 \%$ de las capturas nuevas. De los 20 eventos de trampeo, $O$. longicaudatus fue la especie más capturada en ambientes domésticos (45\% del total).

El número de capturas de roedores varió significativamente entre ambientes según la especie (GLMM; $\chi_{(2)}^{2}=8.29 ; P=0.0158$ ) (Tabla 1). El número de capturas de $O$. longicaudatus en ambos ambientes, como de $A$. hirta en el 
Tabla 1. Número de capturas nuevas en ambiente silvestre y doméstico, número total de capturas nuevas y porcentaje en ambiente doméstico del total de capturas nuevas discriminado por especie. Los números entre paréntesis corresponden a los valores de las variables con la inclusión de las recapturas.

Table 1. Number of new captures in sylvan and domestic environment, number of total new captures, and percentage of the total new captures in domestic environment among species. The numbers in parentheses correspond to the values of the variables with the inclusion of recaptures.

\begin{tabular}{cccc}
\hline & \multicolumn{3}{c}{ Especie } \\
& $\begin{array}{c}\text { O. } \\
\text { longicaudatus }\end{array}$ & $\begin{array}{c}\text { A. } \\
\text { olivacea }\end{array}$ & $\begin{array}{c}\text { A. } \\
\text { hirta }\end{array}$ \\
\hline $\mathrm{N}^{\circ}$ capturas en & 410 & 99 & 444 \\
silvestre (S) & $(473)$ & $(115)$ & $(698)$ \\
$\mathrm{N}^{\circ}$ capturas en & 131 & 39 & 27 \\
doméstico (D) & $(194)$ & $(55)$ & $(41)$ \\
$\mathrm{N}^{\circ}$ total de capturas & 541 & 138 & 471 \\
$\quad$ (S+D) & $(667)$ & $(170)$ & $(739)$ \\
\% en doméstico del & 24.21 & 28.26 & 5.73 \\
total de capturas & $(29.09)$ & $(32.35)$ & $(5.55)$ \\
\hline
\end{tabular}

ambiente silvestre, fue significativamente mayor que en el resto de las combinaciones de especies por ambiente (LSD prueba de Fisher; $P<0.05)$.

La proporción de capturas en los ambientes domésticos difirió significativamente entre especies de roedores $\left(\chi_{(2)}^{2}=48.62 ; P<0.0001\right)$. Oligoryzomys longicaudatus fue capturado dentro del ambiente doméstico más frecuentemente de lo esperado, mientras que las capturas de $A$. hirta en el ambiente silvestre fueron significativamente mayores a lo esperado.

\section{Áreas de acción}

Las áreas de acción promedio no difirieron significativamente entre sexo de los individuos $\left(\right.$ GLMM; $\left.F_{(1,37}=1.45 ; P>0.05\right)$. Sin embargo, el análisis mostró una tendencia en cuanto al efecto de especie sobre el tamaño promedio de las áreas de acción (GLMM; $F_{(1,37)}=2.97$; $P=0.092$ ). Los individuos de $O$. longicaudatus tendieron a menores tamaños promedio de áreas de acción $\left(585.28 \mathrm{~m}^{2}\right.$ ) que los correspondientes a individuos de $A$. hirta $\left(1001.31 \mathrm{~m}^{2}\right)$, independientemente del sexo. De manera general, los tamaños de área de acción para individuos de $O$. longicaudatus oscilaron entre $64.54 \mathrm{~m}^{2}$ y $2704.06 \mathrm{~m}^{2}$, y para A. hirta, entre $71.42 \mathrm{~m}^{2}$ y $4451.06 \mathrm{~m}^{2}$.

\section{Movimientos intra- e inter-ambiente}

Con respecto a los movimientos entre ambos tipos de ambientes (Figura 2), los movimientos

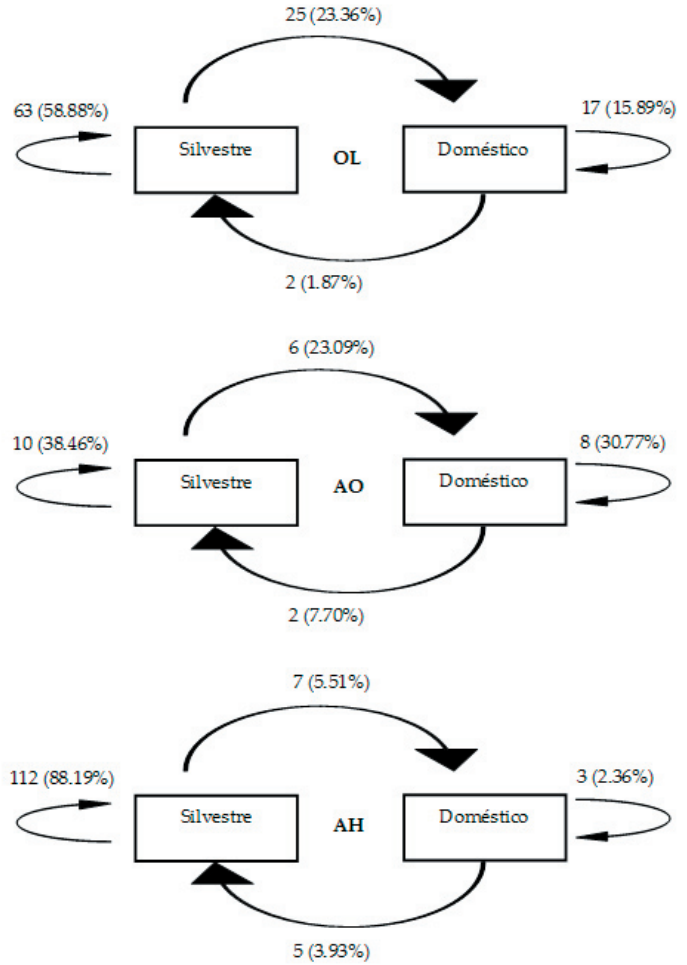

Figura 2. Número de movimientos intra- e inter-ambiente (silvestre y doméstico) para $\mathrm{OL}=O$. longicaudatus, $\mathrm{AO}=A$. olivacea y $\mathrm{AH}=A$. hirta. Entre paréntesis se muestran los porcentajes relativos de cada tipo de movimiento respecto del número total de movimientos.

Figure 2. Number of intra- and inter-environment movements [sylvan=silvestre and domestic $=$ doméstico] for $\mathrm{OL}=O$. longicaudatus, $\mathrm{AO}=A$. olivacea y $\mathrm{AH}=A$. hirta. Between brackets the relative percentages of each type of movement in relation to the total number of movements.

intra-ambiente fueron significativamente más frecuentes que los inter-ambiente para O. longicaudatus (prueba exacta de Fisher; $P$ (bilateral $)<0.0001)$. El $59 \%(\mathrm{n}=107)$ de sus movimientos parten del ambiente silvestre y concluyen en el mismo ambiente y el $89 \%(n=19)$ de los individuos originalmente capturados en el ambiente doméstico permanecen moviéndose en ese ambiente. Sin embargo, $28 \%(n=88)$ de los capturados originalmente en el ambiente silvestre se mueven hacia el ambiente doméstico. Los movimientos de A. olivacea hacia el ambiente de destino son independientes del ambiente desde donde los individuos originaron el movimiento (prueba exacta de Fisher; $P$ (bilateral $)>0.05$ ). En este caso, independientemente del ambiente donde comienza el movimiento, el ambiente destino es indistinto y no difiere del azar, lo que implica un comportamiento de movimientos azaroso tipo "random walk". Finalmente, las capturas en el ambiente de destino para $A$. hirta depende del ambiente original donde 
los individuos fueron capturados (prueba exacta de Fisher; $P($ bilateral $)=0.016)$. En este caso, la mayoría de los movimientos que parten del ambiente silvestre, concluyen en el mismo ambiente (94\%; n=119). Sin embargo, los movimientos que parten del ambiente doméstico, finalizan indistintamente en cualquier ambiente $(\mathrm{n}=8)$. Por lo tanto, para A. hirta los movimientos intra-ambiente (en el ambiente silvestre) son significativamente más frecuentes que los inter-ambiente y que los intra ambiente en el ambiente doméstico.

\section{Discusión}

Oligoryzomys longicaudatus resultó ser una especie muy abundante en ambos ambientes y la más frecuentemente capturada en el ambiente doméstico. Desde el punto de vista sanitario y epidemiológico, su actividad y presencia en el ambiente doméstico es directamente proporcional al riesgo de infección por Hantavirus. El aumento de la densidad de roedores en un ambiente y la competencia por recursos son causas del aumento de la seroprevalencia; esto conlleva a un aumento en el riesgo de infección en ambientes dominados por el ser humano y sus actividades (Armién et al. 2009). Por lo tanto, el riesgo de infección por Hantavirus en humanos es función de la frecuencia de seropositividad y de la abundancia relativa de roedores reservorio en ambientes utilizados por personas, donde el refugio y el alimento no son recursos limitantes (Mills et al. 1997; Glass et al. 2007). Sin embargo, en áreas antropizadas de una zona andina en la Provincia de Chubut, O. longicaudatus mostró baja abundancia, a pesar de ser la especie más frecuentemente capturada en áreas boscosas (Polop et al. 2010).

Por otro lado, el riesgo de infección por Hantavirus en ambientes domésticos podría modularse por la diversidad específica del ensamble de los vectores (Ostfeld and Feesing 2000; Schmidt and Ostfeld 2001; Piudo et al. 2011). En este sentido, el efecto que pueda producir el reservorio principal en un ensamble relativamente biodiverso se 'diluye' y, por lo tanto, se reduce el riesgo de infección en humanos, ya que el escenario ambiental presentaría una cantidad potencial de especies reservorios para un determinado patógeno (en nuestro caso, el ANDV). Bajo esta hipótesis, los ambientes domésticos serían los menos diluidos (i.e., menor riqueza específica) respecto a los silvestres, lo que genera más chances de contagio de Hantavirus (Polop et al. 2010). Algunos estudios en Argentina y Chile también dan cuenta de serología positiva para ANDV en A. hirta, A. olivacea, Loxodontomys micropus y Phyillotis darwini (Pavletic 2000; Cantoni et al. 2001; Larrieu et al. 2008). Sin embargo, hasta la fecha no hay evidencia que involucre a estas especies en la transmisión del virus a humanos, con lo cual se afianza la idea de una dispersión del virus desde su reservorio natural.

La asociación espacial negativa de la distribución de capturas para especies de roedores en agroecosistemas pampeanos podría ser una expresión de la competencia interespecífica por el espacio, lo que causa una segregación interespecífica a nivel de individuo (Busch and Kravetz 1992). Algo análogo podría ocurrir con $O$. longicaudatus, ya que si bien más de $60 \%$ de sus capturas fueron en el ambiente silvestre, es la especie que, en proporción, más usa el ambiente domiciliario, con el mayor porcentaje de capturas durante casi todo el año (>29\%). En este sentido, la competencia interespecífica por el recurso se estaría dando principalmente en el ambiente silvestre, lo cual propicia la segregación y el uso estratégico del ambiente doméstico, con la consecuente reducción de efectos competitivos, posiblemente con $A$. hirta (especie numéricamente dominante) (Piudo et al. 2011).

Los movimientos intra- e inter-ambiente de las tres especies son diferentes, lo que conlleva implicancias sanitarias disimiles. Abrothrix hirta se mueve preferentemente dentro del ambiente silvestre, mientras que $A$. olivacea es un explorador aleatorio del paisaje ("random walk") que usa ambos ambientes de manera indistinta. Sin embargo, O. longicaudatus prioriza los movimientos intra-ambiente de manera significativa $y$, en términos de número de individuos, muestra una mayor circulación relativa desde el ambiente silvestre (ambiente fuente) hacia el doméstico (ambiente sumidero) (ver Pulliam 1988; Pulliam and Danielson 1992). Nuestros resultados coinciden con estudios realizados con tres especies de roedores en Kansas (EE.UU.), en donde las especies realizan más movimientos desde las fuentes hacia los sumideros que viceversa (Diffendorfer et al. 1995). Probablemente, la competencia interespecífica en ambientes cualitativamente buenos puede forzar a los individuos subordinados hacia ambientes subóptimos (Holt 1993), siendo O. longicaudatus la especie subordinada en el ambiente fuente y 'forzada' a dispersar 
al ambiente sumidero minimizando de esta forma los efectos competitivos con las otras especies del ensamble.

Este comportamiento espacial de $O$. longicaudatus posee una relevancia epidemiológica elevada en cuanto al riesgo de contagio de ANDV, ya que al aumentar el número de movimientos inter-ambiente aumenta la probabilidad de asegurar el flujo y la mantención del circuito viral en el ambiente heterogéneo antropizado (Douglass et al. 2006; Lonner et al. 2008; Piudo et al. 2011). Para el caso del ratón ciervo (Peromyscus maniculatus), las diferencias de movimiento de individuos entre distintos tipos de ambiente puede contribuir a una mayor prevalencia de infección con Hantavirus sin nombre (SNV) entre las poblaciones de esa especie. Esto se debe a que los movimientos que conllevan grandes distancias deberían aumentar el número de encuentros casuales con otros individuos y, por lo tanto, el potencial de interacciones intraespecíficas (Root et al. 1999). Por otro lado, en un sitio con mayor abundancia de roedores (como O. longicaudatus en los domicilios en ciertas épocas del año) deberían ocurrir más encuentros casuales, con el consiguiente aumento de ratones infectados con el SNV (Mills et al. 1997). Estos encuentros casuales entre roedores no necesariamente deben ser agresivos para que se produzca la transmisión del virus. Las mordeduras entre roedores sigmodontinos capturados en Chile no serían una condición "sine qua non" para que se produzca la transmisión oral del virus, sino, por contacto, vía saliva o aerosoles de la saliva en encuentros no necesariamente agresivos (Padula et al. 2004). De manera similar, en Montana (EE.UU.) se verificó una falta de correlación entre el contagio del SNV y la presencia de heridas en $P$. maniculatus (Douglas et al. 2007). Es decir que las chances de contagio entre roedores estarían más vinculadas a una combinación del número y tipo de encuentros más que a cada uno de esos factores por separado.

Según estudios previos en la zona, $O$. longicaudatus realiza los desplazamientos más largos entre ambientes, con distancias medias recorridas de más de $30 \mathrm{~m}$. (Piudo 2011; Piudo et al. 2011). Esto incrementa las chances de infectarse con ANDV mediante encuentros con otros individuos potencialmente infectados y también colabora con la circulación del virus entre ambientes, lo que aumenta el riesgo para la salud humana. Por otro lado, algunos estudios sobre el movimiento de individuos en diferentes tipos de ambientes asumen que una mayor distancia de movimiento podría reducir el nivel de competencia intraespecífica e incrementar la posibilidad de encontrar recursos sin explotar, pero también aumentar la exposición de los animales a la depredación (Murrell et al. 2002; Kallimanis et al. 2006). En este contexto, $O$. longicaudatus podría reducir efectos competitivos (i.e., escape competitivo) a través de su habilidad para recorrer grandes distancias.

En los estados de Montana y Nuevo México (EE.UU.), la prevalencia de anticuerpos contra SNV en P. maniculatus en ambientes domésticos es casi dos veces mayor que en ambientes silvestres, ya que las áreas de acción más pequeñas en los ambientes domésticos pueden concentrar la liberación del SNV. Los $P$. maniculatus domésticos se mueven entre las construcciones del domicilio y zonas silvestres, y pueden transmitir el SNV a los individuos que usan ambos ambientes (Douglass et al. 2006). En el presente estudio, $A$. hirta utiliza en forma casi exclusiva el ambiente silvestre, con áreas de acción cercanas al doble en extensión que $O$. longicaudatus. En este escenario, $O$. longicaudatus minimizaría posibles efectos competitivos interespecíficos con $A$. hirta mediante la reducción de sus áreas de acción y el uso de ambos ambientes durante sus actividades. Este comportamiento espacial contribuye a mantener la circulación y el flujo de virus entre estos ambientes y explicaría por qué los individuos de O. longicaudatus de ambientes domésticos poseen 2.44 veces más chances de contener anticuerpos positivos para ANDV que los individuos de zonas silvestres (Piudo et al. 2011). De manera complementaria, en Chile se ha reportado a O. longicaudatus como la única especie de roedor capturada en ambientes domésticos que presentó anticuerpos contra Hantavirus (Torres-Pérez et al. 2004).

A pesar de nuestros resultados, $O$. longicaudatus es considerada una especie de alta vagilidad, con áreas de acción extensas que pueden alcanzar los $4800 \mathrm{~m}^{2}$ (Murúa et al. 1986; Belmar-Lucero et al. 2009; Andreo 2012). Estudios realizados en Chile también describen mayores áreas de acción relativas para esta especie (Agüero and Simonetti 1988; Muñoz-Pedreros et al. 1990). Esto hace particular a nuestro sistema de estudio (ambiente-roedor), ya que el comportamiento espacial de $O$. longicaudatus resultó en menores áreas de acción. Esto podría explicarse por diferencias en la metodología de estimación 
de las áreas de acción y/o por características ambientales de los sitios de muestreo escogidos en cada caso.

La influencia de la dispersión de animales reservorio ha demostrado ser más importante en la transmisión de enfermedades cuando la destrucción, modificación o alteración del ambiente original conlleva un alto porcentaje de pérdida de ambiente original y una baja correlación con el ambiente adyacente (Su et al. 2009; Su 2011). Esto ocurre en el Pje. El Contra, en donde la modificación de los ambientes originales ha dado lugar a los ambientes domésticos bien definidos. Así, los nuevos ambientes a ocupar por O. longicaudatus son principalmente los domésticos cercanos, en particular aquellos donde los roedores residentes son removidos sin previa hermetización de viviendas (Douglass et al. 2003; Piudo 2011). Por lo tanto, los ambientes domésticos son muy riesgosos para los seres humanos porque allí aumentan las chances de contraer Hantavirus; poseen el mayor número de capturas de $O$. longicaudatus a través de movimientos dispersivos inter-ambiente, tienen alta persistencia de esa especie en ese ambiente (i.e., gran número de movimientos intra-ambiente) y albergan el mayor número de individuos de esta especie seropositivos para ANDV en la Provincia de Neuquén (Piudo et al. 2011; este trabajo).

El esfuerzo de muestreo necesario para describir la ecología de un sistema roedorHantavirusesalto, ya queexisten variosfactores que modulan las dinámicas poblacionales del reservorio y de la prevalencia de infección (Douglass and Vadell 2016). En este contexto, estudios a largo plazo de marcado y recaptura de roedores (Mills et al. 2007; Armien et al.
2009; Piudo et al. 2011) resultan relevantes eco-epidemiológicamente, ya que intentan clarificar alguno de esos aspectos como el de la prevalencia de anticuerpos y la ecología espacial de los roedores reservorio en sistemas silvestre-doméstico. Además, permiten modelar evitando las generalizaciones de la extrapolación, lo que los hace marcadamente más útiles en el contexto local (Mills et al. 2010; Douglass and Vadell 2016). De este modo, en el escenario general de nuestra área de estudio, el riesgo de infección por Hantavirus podría reducirse drásticamente mediante la remoción sistemática de roedores en ambientes domésticos con la previa hermetización de las viviendas rurales y una política de ordenamiento territorial local en las construcciones domésticas evitando la entrada y permanencia de nuevos roedores silvestres. Estas medidas de manejo permitirían minimizar la circulación del patógeno entre ambientes y entre individuos reservorios, al menos en el ambiente potencialmente más riesgoso: el doméstico.

Agradecimientos. A Oscar "el Paila" Pailacura por su invaluable ayuda en el campo. A "la Nena" Neira, Doña Aurelia y Franklin Briceño por permitirnos trabajar en sus propiedades. Agradecemos también a la Administración de Parques Nacionales por otorgar los permisos necesarios para llevar a cabo este trabajo. Este trabajo fue parcialmente subsidiado por The National Institute of Health (N P20RR1645505 del programa INBRE-BRIN), por el Center for Disease Control and Prevention a través del acuerdo cooperativo N US3/CCU813599 y por el Centro de Ecología Aplicada del Neuquén. A los tres revisores anónimos por sus aportes a la mejora de este trabajo.

\section{REFERENCIAS}

Agresti, A. 2002. Categorical Data Analysis, Second edition. New Jersey: John Wiley and Sons, Inc.

Agüero, T., and J. A. Simonetti. 1988. Home range assessment: a comparison of five methods. Revista Chilena de Historia Natural 61:223-229.

Andreo, V. C. 2012. Variables ambientales en la dinámica espacial de Oligoryzomys longicaudatus (huésped del virus Andes) en la región Noroeste de Chubut, Argentina. Tesis doctoral. Universidad Nacional de Río Cuarto, Córdoba. Argentina. Pp. 269.

Armién, A. G., B. Armién, F. Koster, J. M. Pascale, M. Ávila, P. González, M. de la Cruz, Y. Zaldivar, Y. Mendoza, F. Gracia, B. Hjelle, S. J. Lee, T. L. Yates, and J. Salazar-Bravo. 2009. Hantavirus infection and habitat associations among rodent populations in agroecosystems of Panamá: implications for human disease risk. American Journal of Tropical Medicine and Hygiene 81(1):59-66.

Avgar, T., A. Mosser, G. S. Brown, and J. M. Fryxell. 2012. Environmental and individual drivers of animal movement patterns across a wide geographical gradient. Journal of Animal Ecology doi:10.1111/j.1365-2656.2012.02035.x.

Belmar-Lucero, S., P. Godoy, M. Ferrés, P. Vial, and R. E. Palma. 2009. Range expansion of Oligoryzomys longicaudatus (Rodentia, Sigmodontinae) in Patagonian Chile, and first record of Hantavirus in the region. Revista Chilena de Historia Natural 82:265-275.

Bowler, D. E., and T. G. Benton. 2005. Causes and consequences of animal dispersal strategies: relating individual behaviour to spatial dynamics. Biological Reviews 80(2):205-225.

Burnham, K. P., and D. R. Anderson. 2002. Model selection and multimodel inference: A practical information-theoretic 
approach. Second edition. Springer-Verlag. ISBN:0-387-95364-7.

Busch, M., and F. O. Kravetz. 1992. Competitive interactions among rodents (Akodon azarae, Calomys laucha, C. musculinus and Oligoryzomys flavescens) in a two-habitat system. I. Spatial and numerical relationships. Mammalia 56(1):45-56.

Cabrera, A., and A. Willink. 1980. Biogeografía de América Latina. Segunda edición. Washington D. C. Secretaría General de la O.E.A. Monografía. No 13. Pp. 122.

Cantoni, G., P. Padula, G. Calderón, J. Mills, E. Herrero, P. Sandoval, V. Martínez, N. Pini, and E. Larrieu. 2001. Seasonal variation in prevalence of antibody to Hantaviruses in rodents from southern Argentina. Tropical Medicine and International Health 6:811-846.

Corbalán, V. E., and R. A. Ojeda. 2005. Áreas de acción en un ensamble de roedores del desierto del monte (Mendoza, Argentina). Mastozoología Neotropical 12(2):145-152.

Diffendorfer, J. E., M. S. Gaines, and R. D. Holt. 1995. Habitat fragmentation and movements of three small mammals (Sigmodon, Microtus, and Peromyscus). Ecology 76(3):827-839.

Douglass, R. J., A. J. Kuenzi, C. Y. Williams, S. J. Douglass, and J. N. Mills. 2003. Removing deer mice from buildings and the risk for human exposure to Sin Nombre virus. Emerging Infectious Diseases 9(3):390-392.

Douglass, R. J., and M. V. Vadell. 2016. How much effort is required to accurately describe the complex ecology of a rodent-borne viral disease? Ecosphere 7(6):1-13:e01368. 10.1002/ecs2.1368.

Douglass, R. J., C. H. Calisher, K. D. Wagoner, and J. N. Mills. 2007. Sin Nombre virus infection of deer mice in Montana: characteristics of newly infected mice, incidence, and temporal pattern of infection. Journal of Wildlife Disease 43:12-22.

Douglass, R. J., W. J. Semmens, S. J. Matlock-Cooley, and A. J. Kuenzi. 2006. Deer mouse movements in peridomestic and sylvan settings in relation to Sin Nombre virus antibody prevalence. Journal of Wildlife Diseases 42(4):813-818.

ESRI. 2011. ArcGIS Desktop: Release 10. Redlands, CA: Environmental Systems Research Institute.

Fahrig, L. 2007. Non-optimal animal movement in human-altered landscapes. Functional Ecology 21(6):1003-1015.

Giuggioli, L., G. Abramson, V. M. Kenkre, R. R. Parmenter, and T. L. Yates. 2006. Theory of home range estimation from displacement measurements of animal populations. Journal of Theoretical Biology 240:126-135.

Glass, G. E., T. Shields, B. Cai, T. L. Yates, and R. Parmenter. 2007. Persistently highest risk areas for Hantavirus pulmonary syndrome: potential sites for refugia. Ecological Applications 17:129-139.

Harris, D. B., and D. W. Macdonald. 2007. Interference competition between introduced black rats and endemic Galapagos rice rats. Ecology 88(9):2330-2344.

Harrison, X. A. 2014. Using observation-level random effects to model overdispersion in count data in ecology and evolution. PeerJ 2:e616; DOI 10.7717/peerj.616.

Hilbe, J. M. 2011. Negative binomial regression. Second edition. Cambridge: Cambridge University Press.

Holt, R. D. 1993. Ecology at the mesoscale: the influence of regional processes on local communities. Pp. 77-88 in R. Ricklefs and D. Schluter (eds.). Species diversity in ecological communities: historical and geographical perspectives. University of Chicago Press. Chicago, Illinois. USA.

Kallimanis, A. S., W. E. Kunin, J. M. Halley, and S. P. Sgardelis. 2006. Patchy disturbance favours longer dispersal distance. Evolutionary Ecology Research 8:529-541.

Kelt, D. A. 2000. Small mammal communities in rainforest fragments in Central Southern Chile. Biological conservation 92:345-358.

Kelt, D. A., M. S. Hafner, and the American Society of Mammalogists Ad Hoc. 2010. Updated guidelines for protection of mammalogists and wildlife researchers from Hantavirus pulmonary syndrome (HPS). Journal of Mammalogy 91(6):1524-1527.

Kelt, D. A., P. L. Meserve, and B. K. Lang. 1994. Quantitative habitat associations of small mammals in a temperate rainforest in southern Chile: empirical patterns and the importance of ecological scale. Journal of Mammalogy 75(4): 890-904.

Kernohan, B. J., R. A. Gitzen, and J. J. Millspaugh. 2001. Radio tracking and animal populations. Pages 125-166 in J. J. Millspaugh and J. M. Marzluff (eds.). Analysis of animal space, use and movements. Academic Press. San Diego, California, USA.

Larrieu, E., E. Herrero, M. García Cachau, J. L. Labanchi, S. Mancini, P. Padula, G. Cantoni, L. Cavagion, E. Álvarez, M. Bruni, S. Albarracín, and O. Arellano. 2003. Seroprevalencia de Hantavirus en roedores y casos humanos en el sur de Argentina. Revista Brasilera de Epidemiología 6:68-75.

Larrieu, E., G. Cantoni, E. Herrero, A. Pérez, G. Talmón, G. Vázquez, O. Arellano, and P. Padula. 2008. Hantavirus antibodies in rodents and human cases with pulmonary syndrome, Río Negro, Argentina. Medicina 68(5):373-379.

Levis, S., S. P. Morzunov, J. E. Rowe, D. Enría, N. Pini, G. Calderón, M. Sabattini and S. c. St. Jeor. 1998. Genetic diversity and epidemiology of Hantaviruses in Argentina. Journal Infectious Diseases 177(3):529-538.

Lin, Y. K., and G. O. Batzli. 2001. The influence of habitat quality on dispersal and population densities of voles. Ecological Monographs 71:245-275.

Lonner, B. N., R. J. Douglass, A. J. Kuenzi, and K. Hughes. 2008. Seroprevalence against Sin Nombre Virus in resident and dispersing deer mice. Vector-Borne and Zoonotic Diseases 8(4):433-442.

López, N., P. J. Padula, C. Rossi, M. E. Lázaro, and M. T. Franze-Fernández. 1996. Genetic identification of a new Hantavirus causing severe pulmonary syndrome in Argentina. Virology 220:223-226.

Meserve, P. L., D. R. Martínez, J. R. Rau, R. Murúa, B. K. Lang, and A. Muñoz-Pedreros. 1999. Comparative demography 
and diversity of small mammals in precordilleran temperate rainforest of southern Chile. Journal of Mammalogy 80(3):880-890.

Mills, J. N., B. R. Amman, and G. E. Glass. 2010. Ecology of Hantaviruses and their hosts in North America. VectorBorne and Zoonotic Diseases 10:563-574.

Mills, J. N., K. Schmidt, B. A. Ellis, G. Calderon, D. A. Enría, and T. G. Ksiazek. 2007. A longitudinal study of Hantavirus infection in three sympatric reservoir species in agroecosystems on the Argentine Pampa. Vector-Borne and Zoonotic Diseases 7:229-240.

Mills, J. N., T. G. Ksiazek, B. A. Ellis, P. E. Rollin, S. T. Nichol, T. L. Yates, W. L. Gannon, C. E. Levy, D. M. Engelthaler, T. Davis, D. T. Tanda, J. W. Frampton, C. R. Nichols, C. J. Peters, and J. E. Childs. 1997. Patterns of association with host and habitat: Antibody reactive with Sin Nombre virus in small mammals in the major biotic communities of the southwestern United States. American Journal of Tropical Medicine and Hygiene 56:273-284.

Mills, J. N., T. L. Yates, J. E. Childs, R. R. Parmenter, T. G. Ksiazek, P. E. Rollin, and C. J. Peters. 1995. Guidelines for working with rodents potentially infected with Hantavirus. Journal of Mammalogy 76(3):716-722.

Moenting, A. E., and D. W. Morris. 2006. Disturbance and habitat use: Is edge more important than area? Oikos 115(1): 23-32.

Monjeau, J. A., R. S. Sikes, E. C. Birney, N. Guthmann, and C. J. Phillips. 1997. Small mammal community composition within the mayor landscape divisions of Patagonia, southern Argentina. Mastozoología Neotropical 4:113-127.

Monteverde, M. J. 2014. Selección de hábitat denso-dependiente y riesgo de exposición al Hantavirus "Andes": un estudio experimental con un ensamble de roedores en Patagonia norte, Argentina. Tesis Doctoral. Universidad de Buenos Aires, Buenos Aires. Argentina. Pp. 205.

Monteverde, M. J., L. Piudo, K. Hodara, and R. J. Douglass. 2011. Population ecology of Eligmodontia morgani (Rodentia, Cricetidae, Sigmodontinae) in northwestern Patagonia. Ecología Austral 21:195-200.

Morris, D. W. 1987. Tests of density dependent habitat selection in a patchy environment. Ecological Monograph 57: 269-281.

Morris, D. W. 1989. Density-dependent habitat selection: testing the theory with fitness data. Evolutionary Ecology 3:80-94.

Morris, D. W. 1991. Fitness and patch selection by white footed mice. The American Naturalist 138:702-716.

Muñoz-Pedreros, A., R. Murúa, and L. González. 1990. Nicho ecológico de micromamíferos en un agroecosistema forestal de Chile central. Revista Chilena de Historia Natural 63: 267-277.

Murrell, D. J., J. M. J. Travis, and C. Dytham. 2002. The evolution of dispersal distance in spatially-structured populations. Oikos 97:229-236.

Murúa, R., L. A. González, and P. L. Meserve. 1986. Population ecology of Oryzomys longicaudatus philippii (Rodentia: Cricetidae) in Southern Chile. Journal of Animal Ecology 554:281-293.

Ostfeld, R. S., and F. Feesing. 2000. The function of biodiversity in the ecology of vector-born zoonotic diseases. Canadian Journal of Zoology 78:2061-2078.

Padula, P., R. Figueroa, M. Navarrete, E. Pizarro, R. Cadiz, C. Bellomo, C. Jofre, L. Zaror, E. Rodríguez, and R. Murúa. 2004. Transmission study of Andes hantavirus Infection in the wild sigmodontine rodents. Journal of Virology 78(21): 11972-11979.

Parmenter R. R., T. L. Yates, D. R. Anderson, K. P. Burnham, J. L. Dunnum, A. B. Franklin, M. T. Friggens, B. C. Lubow, M. Miller, G. S. Olson, C. A. Parmenter, J. Pollard, E. Rexstad, T. M. Shenk, T. R. Stanley, and G. C. White. 2003. Small-mammal density estimation: a field comparison of grid-based vs. web-based density estimators. Ecological Monographs 73(1):1-26.

Parmenter, R. R. and J. A. MacMahon. 1989. Animal density estimation using a trapping web design: Field validation experiments. Ecology 70(1):169-179.

Pavlacky, D. C. Jr., H. P. Possingham, A. J. Lowe, P. J. Prentis, D. J. Green, and A. W. Goldizen. 2012. Anthropogenic landscape change promotes asymmetric dispersal and limits regional patch occupancy in a spatially structured bird population. Journal of Animal Ecology 81:940-952.

Pavletic, C. 2000. Hantavirus: su distribución geográfica entre los roedores silvestres de Chile. Revista Chilena de Infectología 17:186-196.

Pearson, O. P. 1983. Characteristics of a mammalian fauna from forests in Patagonia, Southern Argentina. Journal of Mammalogy 64(3):476-492.

Piudo, L. 2011. Efecto de la modificación antropogénica del hábitat en la composición e infección de roedores y su implicancia en el riesgo de contagio por Hantavirus. Tesis Doctoral. Universidad Nacional del Comahue, Río Negro. Argentina. Pp. 106.

Piudo, L., M. Monteverde, S. González Capria, P. Padula, and P. Carmanchahi. 2005. Distribution and abundance of sigmodontine rodents in relation to Hantavirus in Neuquén, Argentina. Journal of Vector Ecology 30(1):119-125.

Piudo. L., M. J. Monteverde, R. S. Walker, and R. J. Douglass. 2011. Rodent community structure and Andes virus infection in sylvan and peridomestic habitats in northwestern Patagonia, Argentina. Vector-Borne and Zoonotic Diseases 11(3):315-324.

Polop, F. J., M. C. Provensal, N. Pini, S. C. Levis, J. W. Priotto, D. Enría, G. E. Calderón, F. Costa, and J. J. Polop. 2010. Temporal and Spatial Host Abundance and Prevalence of Andes Hantavirus in Southern Argentina. EcoHealth 7(2): 176-184.

Pulliam, H. R. 1988. Sources, sinks, and population regulation. The American Naturalist 132(5):652-661. 
Pulliam, H. R., and B. J. Danielson. 1992. Sources, sinks, and habitat selection: a landscape perspective on population dynamics. The American Naturalist 137:S50-S66.

R Development Core Team. 2014. R: A language and environment for statistical computing. Wien, Austria: R foundation for statistical computing. URL: www.R-proyect.org.

Root, J. J., C. H. Calisher, and B. J. Beaty. 1999. Relationships of deer mouse movement, vegetative Structure, and prevalence of infection with Sin Nombre virus. Journal of Wildlife Diseases 35(2):311-318.

Schmidt, K. A. and R. S. Ostfeld. 2001. Biodiversity and dilution effect. Ecology 82:609-619.

Southwood, T. R. E. 1966. Ecological Methods. Methunen and Co. Ltd. London.

$\mathrm{Su}, \mathrm{M}$. 2011.The effect of dispersal on the population dynamics of a host-parasite system in fragmented landscape. Acta Ecologica Sinica 31(12):3265-3269.

Su, M., C. Hui, Y. Zhang, and Z. Lia. 2009. How does the spatial structure of habitat loss affect the eco-epidemic dynamics? Ecological Modelling 220(1):51-59.

Teta, P., and U. F. J. Pardiñas. 2014. Variación morfológica cualitativa y cuantitativa en Abrothrix longipilis (Cricetidae, Sigmodontinae). Mastozoología Neotropical 21(2): 291-309.

Torres-Pérez, F., J. Navarrete-Droguett, R. Aldunate, T. L. Yates, G. J. Mertz, P. A. Vial, M. Ferrés, P. A. Marquet, and R. Eduardo Palma. 2004. Peridomestic small mammals associated with confirmed cases of human Hantavirus disease in southcentral Chile. Tropical Medicine and International Health 70(3):305-309.

Udrizar Sauthier, D. E., A. Andrade, and U. F. J. Pardiñas. 2005. Predation of small mammals by Rufous Legged Owl, Barn Owl, and Magellanic Horned Owl in Argentinean Patagonia forests. Journal of Raptor Research 39:163-166.

Zheng, C., J. Pennanen, and O. Ovaskainen. 2009. Modelling dispersal with diffusion and habitat selection: analytical results for highly fragmented landscapes. Ecological Modelling 220(12):1495-1505.

Zollner, P. A. 2000. Comparing the landscape level perceptual abilities of forest sciurids in fragmented agricultural landscapes. Landscape Ecology 15:523-533. 